\title{
Usage of Posts
}

\author{
Aishwarya Das ${ }^{1}$, Iffat Nasim ${ }^{2}$ \\ ${ }^{1}$ Department of Conservative Dentistry and Endodontics, Chennai, Tamil Nadu, India. \\ ${ }^{2}$ Department of Conservative Dentistry and Endodontics, Chennai, Tamil Nadu, India.
}

\section{ABSTRACT}

\section{BACKGROUND}

The ideal management of a severely damaged tooth structure with any sort of periapical or pulpal pathology is dependent on a definitive and careful endodontic treatment as well as on a good and strong prosthodontic management which should be tried to be accomplished by all clinicians. Majority of the teeth which have undergone pulp extirpation and subsequent treatment are weak structurally and require an endodontic post and core build system for adequate functionality as well as aesthetics. Amongst all the available options for post and core systems, the decision to select an appropriate one can be challenging to the clinicians. If a tooth has lost significant coronal structure due to underlying caries or any sort of pulpal pathology, it is important to treat it endodontically also equal attention should be paid to its restoration to its anatomic form and function. However when sufficient remaining tooth substance is present, there no need of a post and the tooth can be endodontically treated with a prosthetic crown for its restoration. If a tooth has lost most of its tooth structure, it definitely necessitates the use of a post. The function of a post is to help retain a core which will provide structure and support to the future crown. An endodontic post not only provides strength and support to the remaining crown structure by retaining the core but also to the radicular part of the tooth. The more the tooth structure is preserved, the lower is the risk of catastrophic failures for that tooth. Posts which are pre - fabricated have numerous options from material aspect. This review article emphasizes on the various parameters to be considered before planning an endodontic post for a tooth.

\section{KEY WORDS}

Endodontic Post, Prognosis, Retention, Strength, Structural Integrity
Corresponding Author: Dr. Aishwarya Das. Mahalaxmi Flats, Besant Nagar,

Chennai, Tamil Nadu, India.

E-mail: aishwarya.das@gmail.com

DOI: $10.14260 /$ jemds/2020/598

How to Cite This Article:

Das A, Nasim I. Usage of Posts. J Evolution Med Dent Sci 2020;9 (37):2755-2760, DOI: $10.14260 /$ jemds $/ 2020 / 598$

Submission 09-06-2020,

Peer Review 04-08-2020,

Acceptance 10-08-2020,

Published 14-09-2020.

Copyright (C) 2020 JEMDS. This is an open access article distributed under Creative Commons Attribution License [Attribution 4.0 International (CC BY 4.0)] 


\section{BACKGROUND}

The number of endodontic treatments being carried out routinely is outnumbering the procedures being performed in the past one decade and with better and reliable prognosis. Eventually as a result, the importance of restoring a tooth to its full form and function after endodontic treatment has become very important. A post and core build up system is frequently recommended for most of the endodontically treated teeth to maintain its structural and functional integrity. A sound knowledge about the endodontic posts and its proper usage is absolutely necessary for a successful restoration. Ultimately, to increase the longevity of the tooth, a good core system and final coverage with a prosthetic crown matters a lot. The primary function of a post is to help retain the core material which will eventually retain the crown. The pros and cons of all post systems available with a multitude of designs and materials should be contemplated well and then a decision should be made regarding their usage. Making the appropriate selection can be challenging for the clinicians. Posts were earlier referred to as dowel. In ancient times, wood was used initially as a dowel which would swell up on absorption of moisture and would occupy the canal space well.

Rather than changes in the dentin, the aggressive access cavity designs led to a significant reduction in the strength and fracture resistance of the root canal treated tooth as compared to a sound tooth. ${ }^{1}$ Aggressive access cavity designs can lead to considerable loss of tooth structure. ${ }^{2,3}$ and result in a failed restoration due to increased microleakage along the margins of the restoration. It will also increase the fracture predilection of the root canal treated tooth. Teeth which are indicated for root canal treatment usually have secondary caries or extensive caries or failed existing restorations which cause a significant loss of tooth structure. Studies by Randow and Glantz, ${ }^{4}$ have claimed that once the tooth becomes pulp less, its protective mechanism is compromised and the predilection for fracture increases in such tooth. Fennis et al. ${ }^{5}$ have conducted studies which indicate that pulp treated teeth are more brittle in nature. Hence, results of studies like these indicate that a definitive restoration is a must for long term clinical success of root canal treated teeth. Also, it is a common notion that use of an endodontic post strengthens the endodontically treated tooth which is not true. It serves as a medium for holding the core which holds the final restoration. Retrievability of posts is also an important feature in case of failure and subsequent removal of it. Certain theories like the Proportions, the Conservationist and the Preservationist are followed while using an endodontic post. The preparation of the post space can be challenging at times. Ideally it is said that apically $4-6 \mathrm{~mm}$ of the root filling material be left. This is because of the fact that, in apical $3-5 \mathrm{~mm}$, the maximum apical ramifications are present. Hence, the need to seal all the portals of entry and exit of the canal.

\section{PARAMETERS THAT DETERMINE USAGE OF POST}

\section{Remaining Sound Tooth Substance}

After completion of the root canal treatment, some teeth have good amount tooth structure remaining and others don't. ${ }^{6}$
Following is the prognosis of the tooth in reducing order of the remaining tooth matter-

1) Sufficient Tooth Structure Present Due Conservative Access Cavity Preparation

Under these conditions, a dowel is not required. Usually a composite resin with a good shade match is sufficient for final restoration of the tooth. Such cases have a successful clinical prognosis.

\section{2) Half of the Tooth Structure Remaining}

Very often we encounter such conditions. Such a tooth may not need a dowel if masticatory load is not heavy in this area. The coronal tooth structure can be built up, usually with resin-based composite build-up material, to provide additional retention for the subsequent restoration, such teeth usually display a good clinical prognosis.

3) Less than Half of the Tooth Structure Remaining These situations validate the use of an endodontic post. But the prognosis may not be good enough in long term and this should be informed well to the patient.

4) Minimal Tooth Structure in the Oral Cavity Such teeth can be retained, if the clinician plans orthodontic extrusion of the tooth. Such teeth can be considered for extraction and subsequent placement of an implant. If it is decided to retain such teeth, a post can be used. Even after placing posts, the prognosis of this tooth may not be favourable.

5) Absence of Tooth Structure Coronally

Such teeth should not be retained. Or else orthodontic extrusion can be tried if the tooth has long roots. But the time involved for this procedure is too long and patient compliance also matters unless it is absolutely necessary to retain the tooth.

\section{Quality of Remaining Tooth Structure}

Often, remaining tooth structure has discoloured areas, slightly demineralized areas, cracks and undermined areas. Removal of tooth structure with these characteristics allows a necessary evaluation of the amount of viable tooth structure remaining. After removing the affected areas, the quantity of remaining tooth structure should be evaluated. Posts are often indicated in such clinical situations. ${ }^{7}$

\section{Remaining Adjacent Teeth}

If a root canal treated tooth is present alone without any adjacent teeth, it will be subjected to significant occlusal forces as compared to a tooth that has sound adjacent teeth.[15] When restoring a tooth that has no or few surrounding teeth, it is appropriate to use a post or posts to strengthen the endodontically treated tooth.

\section{Occlusion}

Patient's occlusion is an important parameter to be considered before placement of post. ${ }^{14}$ Patients with bruxing or clenching habits, stress the teeth far more than those patients with 
normal occlusion. Enormous lateral stresses are placed on the teeth of these patients. Hence decision should be taken to place posts in bruxing and clenching patients.

\section{Planned Restoration to Be Placed on the Tooth}

A root canal treated tooth with a single crown has minimal stress when compared to a tooth to be used as an abutment for a fixed prosthesis or a removable partial denture. ${ }^{7}$ If an endodontically treated tooth is expected to have significant load placed on it in service, post should be placed.

\section{Indications for Dowel}

A dowel or post is generally used in cases of root canal treated teeth which have compromised tooth structure. Following are general indications for an endodontic post or dowel:

- In situations where there is considerable (two thirds or more) loss of sound tooth structure.

- When the endodontically treated tooth is to be used as an abutment for fixed partial denture.

- When there is a need to alter the axial plane of the involved tooth which is more than $1 \mathrm{~mm}$.

- For an anterior tooth which is root canal treated to avoid the risk of fracture at the cervical area of the same tooth.

- Where the mesial and distal sides of the tooth are lost, and access cavity has been prepared lingually further weakening the existing tooth structure.

Generally, two categories of posts systems exist, prefabricated and cast posts. Prefabricated posts can be of metal or fiber. They are used commonly because of reduced chairside time and ease of use. Following are the indications of prefabricated posts.

- When the natural tooth structure of an endodontically treated tooth is lost or is extensively damaged.

- When aesthetics is one of primary requirements.

- When a change in the axial position of the tooth is required.

- When the post and core build up needs to be done in a single appointment.

Customized post and core systems were well accepted by most clinicians and are used widely even now. But they needed multiple appointments and a good temporization as it may take time between appointments. Cast posts are of particular help when there is a need of multiple posts in the same arch. Using the same impression, in the lab, multiple posts can be cast and cemented in one appointment rather than individually going for post and core build separately for each tooth. However, it is absolutely important to place a good aesthetic temporary crown in anterior teeth when cast post is planned for them. During the placement of a temporary crown, a barrier material is must over the obturating material and also during cementation of cast post one has to be really quick.

\section{Indications for Customized Cast Post}

- In cases requiring three or more posts in the upper or lower arch.

- In teeth requiring post but having minimal tooth structure.
- In case if change in axial orientation of the tooth is required.

- Cases of all ceramic crown.

- Cases with bulbous or irregularly shaped canals.

\section{Types of Post}

Posts are grouped into a number of ways. Primarily, they are divided into active, passive, parallel, tapered or based on composition of materials used.

\section{Active and Passive Posts}

The primary reason for better retention of the active posts are due to the inherent threads in them which very well engage in the canal walls and obtain anchorage. In contrary to them, the parallel posts rely on the luting agent for their anchorage. Active posts are better retentive but induce additional stresses in the radicular portion of the tooth as compared to tapered ones. But if we have good remaining dentinal thickness, active posts are a good choice. However they should be avoided in teeth which have short root.

\section{Parallel and Tapered Posts}

On being compared, parallel posts are retained better than tapered posts. The former exert considerably lower stresses on the radicular part of the tooth, as the wedging stresses are much lower in parallel posts which lowers the risk of tooth fracture as compared to the tapered posts. Studies by Sorenson and Martin off have reported greater success in case of parallel posts as compared to tapered ones. On the other hand, tapered are generally indicated for teeth with narrow roots and also these posts require lesser dentin removal as the shape of most roots are in generally tapered.

\section{Type of Metal Used}

Stainless steel, nickel chromium, or titanium are generally used for fabrication of prefabricated metal post systems. These posts are known to be very strong and tough with the exception of titanium alloys. Owing to their shape which is round, they offer quite less resistance to rotational forces. If sufficient tooth structure remains, there is no issue but if remaining tooth structure is less, anti-rotational features need to be incorporated in the form of pins and slots. As far as the core is concerned, a bonded material is needed.

Tapered and passive posts offer less retention but they ensure minimal dentin removal as their shape resembles that of the canal. If at least $8 \mathrm{~mm}$ root length is available, they can be suitably used in thin roots like those of premolars. Active posts and usage of resin cements can provide additional retention.

Mostly titanium is used for manufacturing of prefab dowels. Corrosion was a concern for other metallic posts hence titanium posts were introduced. Titanium posts have radio opacity similar to gutta percha. Titanium posts can't be used in thin root canals as they have significantly lower fracture resistance. Retrieval of titanium posts can result in their breakage at times as they are fragile. Ultrasonic agitation can be used for their retrieval but that may cause injury to the 
tooth and surrounding tissues. Because of the above reasons, usage of titanium and brass posts is not encouraged.

Customized posts and prefabricated posts are the major categories of posts to be used by clinicians. Customized posts have shown good clinical success when used for root canal treated teeth. Cast posts showed a success of $90.6 \%$ when used as a foundation restoration. ${ }^{8}$ Usually nickel chromium is used as they have good compressive strength and can resist heavy masticatory load.

\section{CLASSIFICATION OF POSTS}

\section{According to DCNA}

1. Metallic

- Custom cast posts.

- Prefabricated.

2. Non-Metallic

- Carbon fiber posts.

- Zirconia posts.

- Woven fiber composite post.

\section{Ingle Classification}

- Custom cast posts.

- Prefabricated posts.

- Tapered, smooth sided posts.

- Parallel sided posts.

- Tapered, self-threading screws.

- $\quad$ Parallel sided, threaded posts.

- $\quad$ Parallel sided, tapered apical end posts.

\section{Barry et al Classification}

1. Active posts

- Parallel sided post positioned by tapping.

- Parallel self-threading.

- $\quad$ Tapered self-threading.

2. Passive posts

- Parallel smooth or serrated.

- Tapered smooth or serrated.

Even now for the longevity of the root canal treated anterior tooth many endodontists prefer a customized cast post. The newer all ceramic materials available can show up the metal underneath hence aesthetics becomes a major concern. Hence a porcelain fused to metal system can be used. ${ }^{9}$ Customized cast posts also require additional appointments as compared to prefabricated posts system hence are more time taking. For long term success of the tooth, customized posts are better because the interfaces involved are less compared to a fiber reinforced post. Hence the chances of failure are more where more interfaces are involved. A study conducted by Rolf $\mathrm{KC}$ et al, concluded that the posts like parallel ones are less retentive but exerts least forces on the root of the tooth. ${ }^{10}$ Active posts provide better retention, but they exert more stress on the root and ideally should be used in short and thin root canals. Pontius and Hutter said that the bonding protocol should be followed well for good retention of the fibre reinforced posts. ${ }^{11}$ If we compare tapered and parallel posts, tapered posts require less removal of root dentin as compared to parallel posts and can be easily used for thin root canals.

DETERMINANTS FOR SELECTION OF POSTS

\section{Length of the Root}

The ideal length of the endodontic post depends on the remaining length of the root and also on its internal shape. ${ }^{12}$ Apically around $3 \mathrm{~mm}$ to $5 \mathrm{~mm}$ of root canal filling material should be left to block the portals of entry and exit to the canal which will help maintain the apical seal.13,14 According to the findings of a study, good luting agents can be used in case the length of the dowel is short. ${ }^{15}$ As we know molars have short roots for increased anchorage and strength we can use two posts. ${ }^{4}$ The longer the length of the root the better the retention of the post. Adequate isolation is also mandatory.

\section{Tooth Anatomy}

Anatomic variations present in the tooth play a major role in deciding about the post. Before planning for the post space preparation, intra oral periapical image of the tooth should be understood well and sound knowledge of root anatomy is a must. Gutmann found that the roots of teeth in maxillary incisors and mandibular premolars are suitable for majority of the available dowels. ${ }^{7}$ It is difficult and unpredictable to place posts in curved canals. It will attract more shear stresses and can lead to fracture of the tooth.

\section{Width of the Dowel}

The breadth of the post is supposed to be less than or equal to a third of the breadth of the radicular part of the tooth ${ }^{16}$ according to the proportionist theory which can preserve sufficient tooth structure. According to the preservationist theory, the dowel should have at least $1 \mathrm{~mm}$ of sound tooth structure around itself. ${ }^{17}$ According to conservationist theory, the radicular part should be prepared as less as possible and the residual dentin should be maintained. 18

\section{Ferrule}

The remaining tooth structure circumferentially above the cervical part of the tooth is very important as it decides the strength of the tooth. ${ }^{19}$ Ideally at least 1.5 to $2 \mathrm{~mm}$ of tooth structure should be there.

\section{Forces}

Teeth which have undergone root canal treatment with an endodontic post are exposed to a lot of forces amongst which shear stresses are not conducive. ${ }^{20}$

\section{Preparation of Post Space}

After deciding about the endodontic post to be used, the next important step is to prepare an accurate post space to receive the post. 


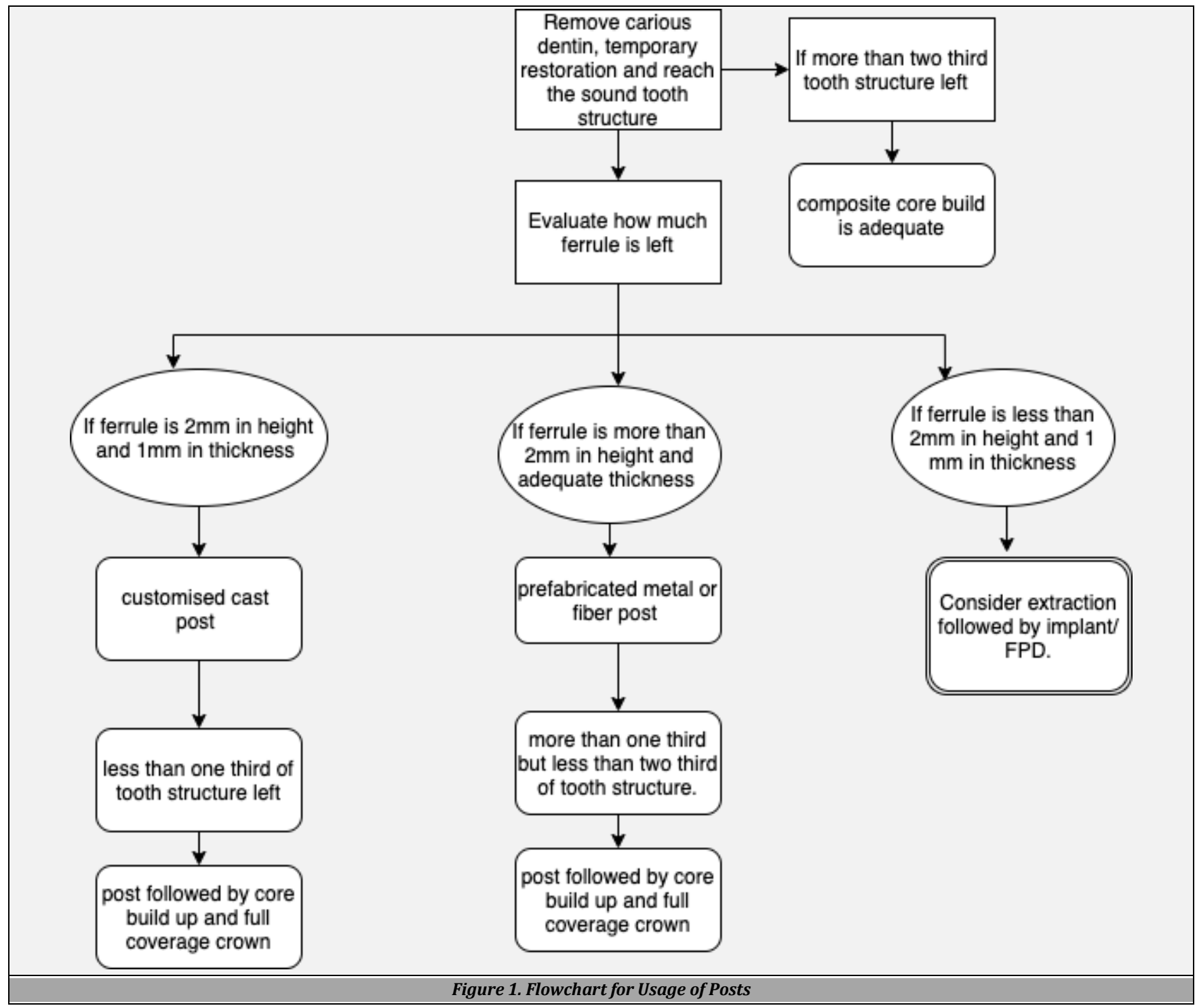

While performing the cleaning and shaping of the root canal, a fair idea about the canal morphology is obtained by the endodontist, which should be kept in mind during post space preparation. The primary goal should be to preserve as much as radicular dentin as possible as this will impart strength to the tooth and increase its fracture resistance. Post space should be ideally two thirds of the root canal space. Maximum stresses are concentrated near the cervical part of the tooth during post placement hence it is recommended that the endodontic post should reach beyond the crestal level of bone. Many methods can be employed for removal of the gutta percha from the canal like heat application, chemicals like gutta percha solvents etc. but most commonly Peeso reamers are used for post space preparation. The gutta percha should be flattened well and the post space in the canal should be dried adequately with paper points to ensure a clean and dry canal space before placement of the post.

\section{Cementation of Post}

For the purpose of cementation of the post, any of the available luting cements like zinc phosphate, glass ionomer, resin cement or resin modified glass ionomer can be used. Though resin cement offers the best retention amongst all it is highly technic sensitive to use. It requires dry canal with the canal space being etched with phosphoric acid, application of bonding agent then using resin cement for cementation. But most of the times it is difficult for the etchant, bonding agent to reach all parts of the post space preparation. This may lead to adhesive failures in future. But if all steps are followed properly, resin cements are one of best luting agents for endodontic posts.

\section{Core Material}

The choice of core material is important, and it retains the future crown. It should exhibit good adhesion to the tooth as well as to post and overlying crown. Composite resins are the material of choice usually. The core build-up should be strong enough to resist the occlusal forces. It should not be seen through the crown hence core build up materials like amalgam are avoided.

\section{Crown}

The choice of crown is made by the clinician in consultation with the patient. It can be all ceramic or metal ceramic. All metal crowns are avoided due to aesthetic reasons. 


\section{CONCLUSIONS}

With so many available choices for posts, the clinician should evaluate the individual tooth and then only select an appropriate post system. The above factors should be well considered before selecting a post system. This will help in successful restoration of most endodontically treated teeth.

Financial or Other Competing Interests: None.

\section{REFERENCES}

[1] Reeh ES, Messer HH, Douglas WH. Reduction in tooth stiffness as a result of endodontic restorative procedures. J Endod 1989;15(11):512-6.

[2] Panitvisai P, Messer HH. Cuspal deflection in molars in relation to endodontic and restorative procedures. J Endod 1995;21(2):57-61.

[3] Gutmann JL. The dentin-root complex: anatomic and biologic considerations in restoring endodontically treated teeth. J Prosthet Dent 1992;67(4):458-67.

[4] Randow K, Glantz PO. On cantilever loading of vital and non-vital teeth $\mathrm{n}$ experimental clinical study. Acta Odontol Scand 1986;44(5):271-7.

[5] Fennis WMM, Kuijs RH, Kreulen CM, et al. A survey of cusp fractures in a population of general dental practices. Int J Prosthodont 2002;15(6):559-63.

[6] Hirshfeld Z, Stern N. Post and core- the biomechanical aspect. Aust Dent J 1972;17(6):467-8.

[7] Gutmann JL. The dentin- root complex: anatomic and biologic considerations in restoring endodontically treated teeth. J Prosthet Dent 1992;67(4):458-67.

[8] Bergman B, Lundquist P, Sjögren U, et al. Restorative and endodontic results after treatment with cast posts and cores. J Prosthet Dent 1989;61(1):10-5.
[9] Hochstedler J, Huband M, Poillion C. Porcelain-fused-tometal post and core: an esthetic alternative. J Dent Technol 1996;13(8):26-9.

[10] Rolf KC, Parker MW, Pelleu GB. Stress analysis of five prefabricated endodontic dowel designs: a photoelastic study. Oper Dent 1992;17(3):86-92.

[11] Pontius O, Nathanson D, Hutter JW, et al. Survival rate and fracture strength of incisors restored with different post and core systems and endodontically treated incisors without coronoradicular reinforcement. J Endod 2002;28(10):710-5.

[12] Holmes DC, Diaz-Arnold AM, Leary JM. Influence of post dimension on stress distribution in dentin. J Prosthet Dent 1996;75(2):140-7.

[13] Mattison GD, Delivanis PD, Thacker RW Jr, et al. Effect of post preparation on the apical seal. J Prothet Dent 1984;51(6):785-9.

[14] Kvist T, Rydin E, Reit C. The relative frequency of periapical lesions in teeth with root canal -retained posts. J Endod 1989;15(12):578-80.

[15] Nissan J, Dmitry Y, Assif D. The use of reinforced composite resin cement as compensation for reduced post length. J Prosthet Dent 2001;86(3):304-8.

[16] Stern N, Hirshfeld Z. Principles of preparing endodontically treated teeth for dowel and core restorations. J Prosthet Dent 1973;30(2):162-5.

[17] Halle EB, Nicholls JI, Van Hassel HJ. An in-vitro comparison of retention between a hollow post and core and a custom hollow post and core. J Endod 1984;10(3):96-100.

[18] Pilo R, Tamse A. Residual dentin thickness in mandibular premolars prepared with gates glidden and Parapost drills. J Prosthet Dent 2000;83(6):617-23.

[19] Barkhordar RA, Radke R, Abbasi J. Effect of metal collars on resistance of endodontically treated teeth to root fracture. J Prosthet Dent 1989;61(6):676-8.

[20] Rosenstiel SR, Land MF, Fujimoto J. Contemporary fixed prosthodontics. $3^{\text {rd }}$ edn. New Delhi: Harcourt (India) Pvt. Ltd., 2001: p. 273-82. 